(C) 2021 IEEE. Personal use of this material is permitted. Permission from IEEE must be obtained for all other uses, in any current or future media, including reprinting/republishing this material for advertising or promotional purposes, creating new collective works, for resale or redistribution to servers or lists, or reuse of any copyrighted component of this work in other works.

\title{
Modeling Snow Losses in Photovoltaic Systems
}

\author{
Mari B. Øgaard ${ }^{1,2}$, Heine N. Riise ${ }^{2}$, and Josefine H. Selj ${ }^{1,2}$ \\ ${ }^{1}$ Department of Technology Systems, University Of Oslo, 2007 Kjeller, Norway \\ ${ }^{2}$ Solar Power Department, Institute for Energy Technology, 2007 Kjeller Norway
}

\begin{abstract}
To achieve accurate PV energy yield assessments for cold climate locations where snowfalls can lead to significant power losses in the wintertime, improvement and validation of snow loss modeling is necessary. In this paper we introduce a snow depth dependent clearing rate coefficient in the Marion snow loss model to account for the effect of different snow conditions on the natural snow clearing process. To evaluate the model, seven roof mounted PV plants with two different system configurations are analyzed. For both system configurations the snow depth dependent clearing rate coefficient yields lower error in the total modeled snow loss and capture climatic variations between locations better compared to the standard constant coefficient.
\end{abstract}

Keywords - photovoltaic systems, performance evaluation, snow, snow loss modeling, soiling

\section{INTRODUCTION}

As cost reductions have made photovoltaics (PV) a favorable choice for electricity generation also in colder climates, deployment rates in regions with snow falls are rapidly increasing [1]. Snow coverage on PV modules will lead to significant power losses. For certain locations snow fall can result in zero electricity production in the winter season and more than $30 \%$ annual losses [2]. Consequently, it is an important parameter to consider in PV system models. Recent research has demonstrated that the uncertainty in energy yield assessments [3-5] and forecasting [6] can be reduced if snow loss models are included. Accurate snow loss modeling is challenging, because the parameters influencing the snow cover and resulting PV system loss are manifold. The influential parameters range from weather conditions (irradiance, temperature, wind, etc.), to installation and technology specific configurations (tilt, module technology/orientation, objects obstructing snow sliding etc.) [7, 8] and type of snow [2]. Multiple snow loss models have been suggested [2], but validation is typically lacking [4].

In our previous work [9], we show that the snow loss model suggested by Marion et al. [3] where snow cover and losses are estimated by using empirical correlations to predict 1) when natural snow clearing of the PV modules occur and 2) how fast the snow is cleared off the modules, performs better than empirical models where a model is built by directly relating snow power loss to system and weather data. For the analyzed system, where modules are installed with a low tilt on a flat roof, we also show that for thin snow covers, the natural snow clearing rate is faster compared to thicker covers. By including this effect in the Marion model, reduced uncertainty in modeled snow losses is achieved.

For models with empirical coefficients estimated from one dataset, it is especially important to validate the model with the same coefficients for other systems with similar system configurations to assure model transferability. In this paper, we validate the results presented in [9] and evaluate the Marion model with snow depth dependent clearing rate coefficients on $\mathrm{PV}$ installations in different regions of Norway, with different climatic conditions. We assess two different system configurations: residential systems on tilted roofs and largescale systems installed with low tilts on flat roofs.

\section{METHODOLOGY}

\section{A. PV system data}

Seven PV installations in Norway, divided between two different system configurations, are analyzed. Three of the systems are residential installations on tilted roofs with modules installed in portrait orientation, and four systems are commercial large-scale systems where the modules are installed in landscape orientation with a low tilt and east/west orientation on flat roofed buildings. Low tilt and east/west orientation are not optimal for total annual production in Norway, but is commonly used on flat roofed buildings to increase the packing density and reduce the seasonality of the production profile. Apart from tilt for the residential systems, the installations with the same system configuration are technically identical. The analyzed installations are situated in three different geographic regions in Norway and have a total installed capacity of $1.6 \mathrm{MW}_{\mathrm{p}}$. The modules of all the systems are crystalline silicon. Geographic 
region in Norway, tilt, Köppen-Geiger (KG) climate zone [10], and length of analysis period for each system is given in Table I. Three different climate zones are represented: Humid continental climate (Dfb), subarctic climate (Dfc) and oceanic climate $(\mathrm{Cfb})$. The variation in snow depth for the four different combinations of geographic region and climate zone is shown for two years in Fig. 1.

TABLE I. ANALYZED PV SYSTEMS

\begin{tabular}{|l|l|l|l|l|}
\hline System ID & Region & Tilt & Climate zone & Analysis period \\
\hline \multicolumn{5}{|c|}{ Residential systems } \\
\hline R1 & Central & 26 & Dfb & Jan 2019 - June 2021 \\
\hline R2 & Central & 24 & Dfc & Jan 2018 - June 2021 \\
\hline R3 & Central & 40 & Dfb & Jan 2019 - June 2021 \\
\hline \multicolumn{5}{|c|}{ Commercial systems } \\
\hline C1 & East & 10 & Dfb & Jan 2015 - Sept 2019 \\
\hline C2 & East & 10 & Dfb & Jan 2017 - Sept 2019 \\
\hline C3 & Central & 10 & Dfb & Jan 2018 - Sept 2019 \\
\hline C4 & West & 10 & Cfb & Jan 2018 - Sept 2019 \\
\hline
\end{tabular}

The production data are collected from the inverters. Snow depth and snow fall data are collected from seNorge.no [11] and temperature and global horizontal irradiation (GHI) data are collected from nearby weather stations [12]. For the commercial system, the effective in plane irradiance is measured by reference cells, and the module temperature is measured by sensors attached to the rear side of the modules. The residential systems have no on-site sensors.

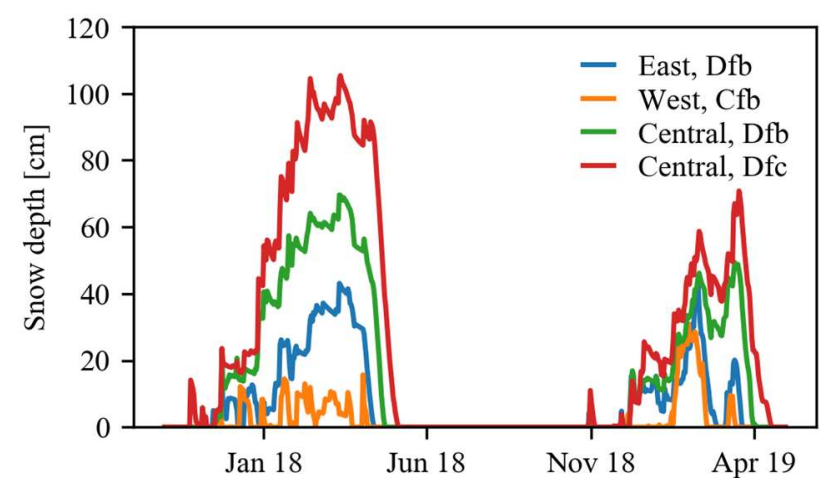

Fig.1. Two years of snow depth data for the four combinations of geographic region and $\mathrm{KG}$ climate zone in the analyzed dataset.

\section{B. Estimating PV power loss}

To estimate the power loss in the PV plants caused by snow, the measured power is compared to models of the systems. The installed capacity, module temperature coefficient, effective irradiance and module temperature are used as inputs to pvlib python [13] where PVWatts is used to model expected power output form the modules. For the commercial systems, measured effective irradiance and module temperature are used as input to the PVWatts model. For the residential systems, effective irradiance and module temperature is modeled from measurements of GHI and ambient temperature by using decomposition, transposition, incident angle modifies and temperature models available in pvlib. By comparing the modeled power to the measured power under snow free conditions, the constant losses of the systems, as well as losses depending on angle of incidence was found. The losses dependent on angle of incidence (AOI) was modeled with the ASHRAE IAM model $[13,14]$ with adjustment parameters fitted to the data. To model the system output, the modeled module power was multiplied with the constant and AOI dependent losses. To estimate the energy loss for different time resolutions, the measured and modeled power was used to calculate the energy on the given time resolution, and the difference in measured and modeled energy was calculated.

As the reference cells can be covered by snow, the measurements of effective irradiance were controlled and corrected by the external GHI data, where the risk of snow cover is lower. Snow cover on the irradiance sensors can lead to underestimation of snow losses. Low irradiance intensity and high incidence angles also contribute to increased modelling uncertainty in the wintertime. If the losses caused by low irradiance intensity and high incidence angles are not properly accounted for in the PV modeling, this can lead to overestimation of snow losses. Because this uncertainty increases with low irradiance, this will not necessarily lead to significant overestimation in absolute losses. For the residential systems, increased modeling uncertainty is also expected because effective irradiance and module temperature is not measured directly at the systems. The total uncertainty in estimation of snow losses is, however, expected to be low compared to the size of the snow losses.

\section{Modeling PV snow loss}

The Marion model initially estimates the snow cover, and subsequently calculates the snow loss based on the snow cover estimate. The model assumes that when the snow starts to melt, it is cleared by sliding off the modules. Direct melting on the modules and snow sliding is thus assumed to occur under the same conditions. Snowfall data are used to identify the presence of snow, and irradiance and module temperature are used to identify conditions where snow melts and slides off the modules. Snow melting and sliding is assumed to happen when:

$$
T_{\mathrm{amb}}>G_{\mathrm{POA}} / m
$$

where $T_{\mathrm{amb}}$ is the ambient temperature, $G_{\mathrm{POA}}$ is the in plane irradiance and $m$ is an empirically defined value of $-80 \mathrm{~W} /\left(\mathrm{m}^{2}\right.$ ${ }^{\circ} \mathrm{C}$ ). How much the snow will slide (or melt), measured in fractions of the total row height, is determined by the tilt of the modules and an empirical snow clearing coefficient $(s c)$ :

$$
\text { Snow slide amount }=s c * \sin (\text { tilt }) \text {. }
$$

From this, the snow coverage on the modules is estimated. The snow loss is subsequently estimated from the calculated snow coverage and the number of parallel connected strings along the row height. The number of parallel connected strings include module substrings to take into account whether the modules are installed in portrait or landscape orientation [12]. If a string is partially covered by snow, the power output is assumed to be zero. The pvlib python implementation of the 
Marion model was used to model the relative snow loss. To estimate the absolute energy loss, the relative snow loss estimated from the Marion was multiplied with the modeled energy output of the system.

For roof mounted systems Marion et al. found $s c$ to be 0.20 [3], which is set to be the default $s c$ in the implementation of the model in pvlib python [13] and the PV modeling software SAM [4]. This coefficient is, however, expected to depend on different system and module designs [9], because technical aspects can either promote or obstruct natural snow clearing [8]. Frameless modules [15], empty space below modules [7], and heating on the rear side of the module [16] will promote sliding, for instance. To our knowledge, $s c$ values for other system designs than the one initially evaluated by Marion et al. are not estimated. As previously mentioned, the rate of snow clearing can also be influenced by the thickness of the snow cover [9]. For thin snow covers snow clearing by direct melting on the module is possible, a process that can be faster than sliding. We therefore use a snow depth dependent $s c$, to include the effect the snow conditions on the natural snow clearing process. Previously, for one of the commercial systems (C1) we found $s c$ to be 0.06 for snow depths larger than $3 \mathrm{~cm}$, and 0.40 for snow depths less than $3 \mathrm{~cm}$ [9]. With the low tilt, and modules installed with no extra elevation above the roof, the snow clearing process is slow during thick snow covers. The interference from the roof reduces the possibility for snow sliding, and snow clearing depends more on direct melting on the modules. The coefficients found for $\mathrm{C} 1$ are evaluated for all the commercial systems in this study, as they all have the same system configurations. For the residential system, the modeled snow loss using varying sliding coefficients are compared to measured losses to estimate the optimal snow clearing coefficients for the three systems.

\section{RESULTS}

\section{A. Flat roof BAPV systems with low tilt modules}

Fig. 2 shows the measured and modeled losses for the two commercial systems located in Eastern Norway. Both of the systems are located on the same location, but on different roofs. We see how the default pvlib $s c$ value $(0.20)$ typically underestimates the losses for the first system, and that a much lower $s c$ of 0.06 gives a better fit between measured and modeled losses for most years. The exception is the results from 2017, a year with very little snow, where the model overestimates the total losses. As previously discussed, the error in total modeled losses for $\mathrm{C} 1$ is reduced if we use separate $s c$ for thin and thick snow covers [9]. The snow depth on the ground is used as an indicator of module snow cover thickness. We see, however, that the measured loss of $\mathrm{C} 2$ is lower than $\mathrm{C} 1$, giving a larger difference between measured and modeled losses for $\mathrm{C} 2$.

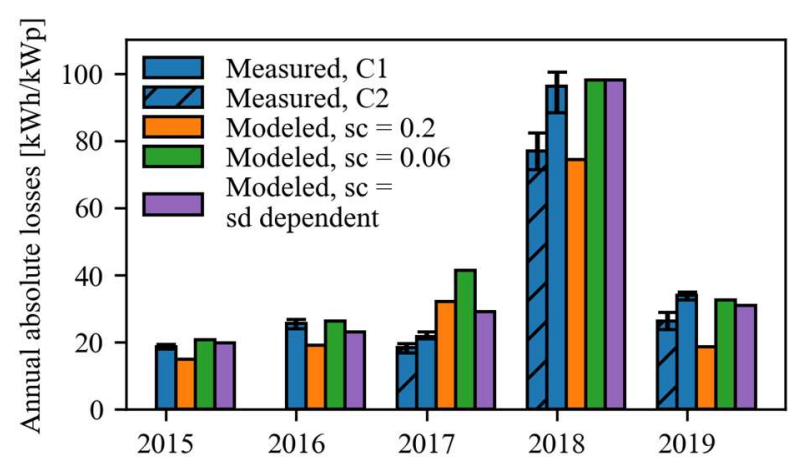

Fig. 2. Measured and modeled annual absolute snow loss for the two commercial systems located in Eastern Norway. The error bar shows the range of the measured losses in the PV plants. The snow loss is modeled with varying $s c$ : 0.2 (default in pvlib), 0.06 , and a snow depth $(s d)$ dependent $s c$, where $s c=$ 0.06 for $s d$ larger than $3 \mathrm{~cm}$ and 0.40 for $s d$ less than $3 \mathrm{~cm}$.

In Fig. 3 the daily losses for $\mathrm{C} 1$ and $\mathrm{C} 2$ in a melting period is shown. We see that the losses of $\mathrm{C} 2$ are reduced faster than for $\mathrm{C} 1$, indicating faster snow melting. The technical configuration, tilt and orientation of the system is the same, the main difference between the two systems is that they are installed on the roofs of different buildings. Here, differences in the heat leakage of the building may explain the deviations. The building where $\mathrm{C} 1$ is installed is expected to have less heat leakage through the roof than the building where $\mathrm{C} 2$ is installed. Heating from below, would naturally aid the snow melting in general, and as previously mentioned also increase the $s c$ of the system. From this comparison, we also observe that discrepancies in the snow loss modelling during springtime, when the irradiance and potential production is high, will contribute greatly to the difference in absolute measured and modeled losses.

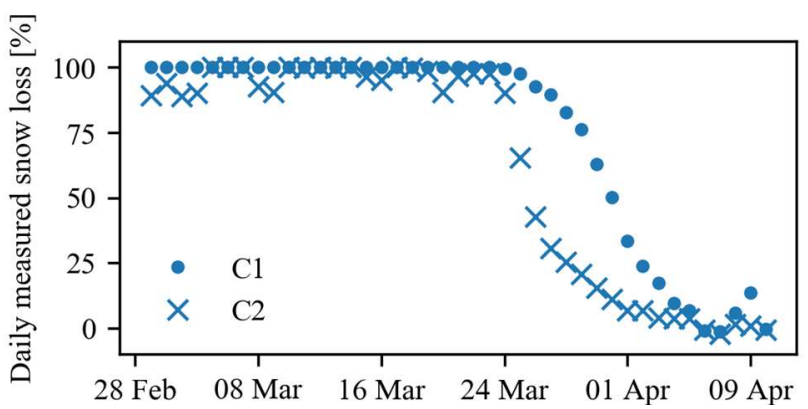

Fig. 3. Measured daily relative loss for the two systems installed at the same location in Eastern Norway, but on different roofs.

For the commercial system located in Central Norway, experiencing much larger snow falls than the other commercial systems (Fig. 1), we observe a new snow depth dependent effect. For very large snow depths, the low tilt of the modules will lead to situations where the snow depth is higher than the system height and the whole system is fully covered by snow. In such situations, we will not expect the PV system to induce any extra snow clearing caused by the tilt or transmitted irradiance heating the module. As long as we have snow depths that are larger than the height of the module above the roof, we therefore set the $s c$ to 0 . For this system, the maximum height of the modules above 
the ground, is approximately $30 \mathrm{~cm}$. Including this in the model, gave a better fit between measured and modeled losses, as shown in Fig. 4.

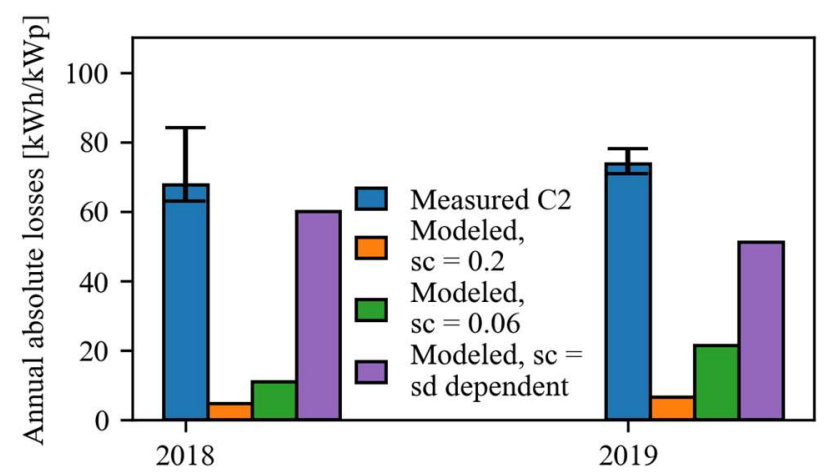

Fig. 4. Measured and modeled annual absolute snow loss for the commercial system located in Central Norway. The error bar shows the range of the measured losses in the PV plant. The snow loss is modeled with varying $s c$ : 0.2 (default in pvlib), 0.06, and a snow depth ( $s d$ ) dependent $s c$, where $s c=0$ for $s d$ larger than $30 \mathrm{~cm}, 0.06$ for $s d$ larger than $3 \mathrm{~cm}$ and 0.40 for $s d$ less than $3 \mathrm{~cm}$.

For the commercial system in Western Norway, which is located on the Norwegian coast in an area with oceanic climate and significantly less snow than the other systems, we see that the losses typically are overestimated (Fig. 5). Together with the results from 2017 at the location in Eastern Norway, this indicates that low snow depths and thin snow covers should be given more consideration in the model.

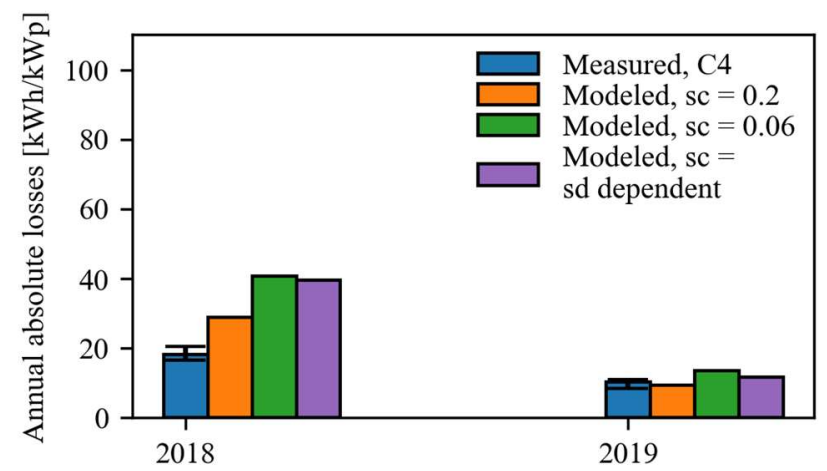

Fig. 5. Measured and modeled annual absolute snow loss for the commercial system located in Western Norway. The error bar shows the range of the measured losses in the PV plant. The snow loss is modeled with varying $s c$ : 0.2 (default in pvlib), 0.06 , and a snow depth $(s d)$ dependent $s c$, where $s c=0.06$ for $s d$ larger than $3 \mathrm{~cm}$ and 0.40 for $s d$ less than $3 \mathrm{~cm}$.

\section{B. BAPV systems on tilted roofs}

All the residential systems are located in Central Norway, but as shown in Table 1 we here have some variation in tilt, and two of the systems are located in areas with humid continental climate, and one of the systems are located in an area with subarctic climate. In Fig. 6-8 the measured losses are shown together with modeled losses using varying snow clearing coefficients. As for the commercial systems, we see that for some years a high $s c$ gives a good fit with the measured losses, and other years a low $s c$ is more suitable. Also here, it is observed that this variation typically is connected to the snow conditions, and that to obtain a general sliding coefficient that gives a satisfactory for all systems and all years, snow depth dependency has to be introduced in the sliding coefficient. The PV modules are not installed at the edge of the roof, and the increased friction of the roof tiles compared to the PV modules is expected to introduce some roof interference. The roof interference is, however, not expected to be as large as for the commercial systems, and with the higher tilt of the residential systems more sliding is expected. Snow depth on the ground is thus not expected to be a good indicator of snow cover thickness. To give an indication of the snow cover thickness on the modules, the accumulated snowfall in the periods with estimated snow losses is calculated and used for the snow depth dependent snow clearing coefficient.

To obtain more accurate results on expected snow clearing coefficients for this system design, more accurate modeling, e.g. by using input data from on-site sensors, or more data is needed.

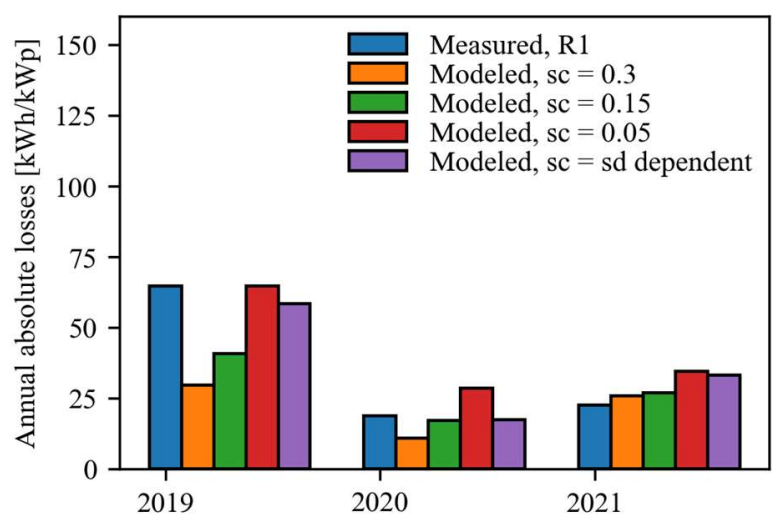

Fig. 6. Measured and modeled annual absolute snow loss for the residential system with a tilt of $26^{\circ}$, located in an area with humid continental climate. The snow loss is modeled with varying $s c$ : $0.3,0.15,0.05$, and a snow depth $(s d)$ dependent $s c$, where $s c=0.05$ for $s d$ larger than $3 \mathrm{~cm}$ and 0.30 for $s d$ less than $3 \mathrm{~cm}$.

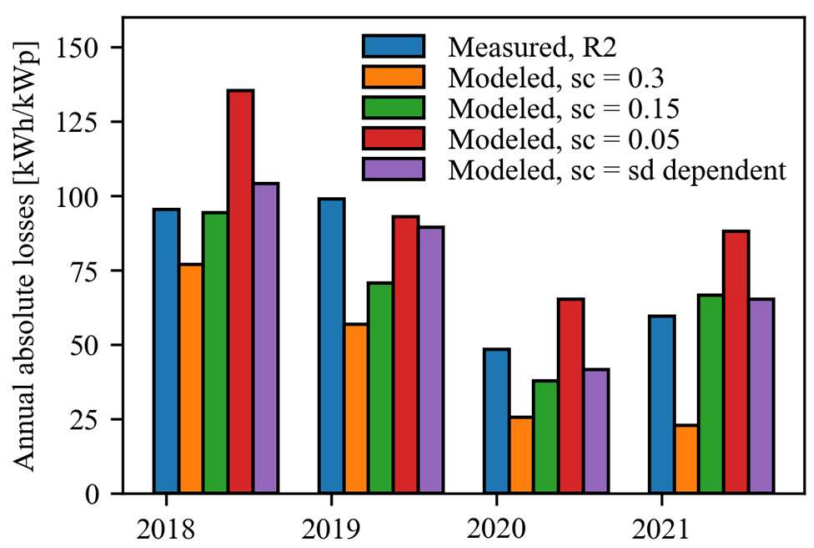

Fig. 7. Measured and modeled annual absolute snow loss for the residential system with a tilt of $24^{\circ}$, located in an area with subarctic climate. The snow loss is modeled with varying $s c$ : $0.3,0.15,0.05$, and a snow depth $(s d)$ 
dependent $s c$, where $s c=0.05$ for $s d$ larger than $3 \mathrm{~cm}$ and 0.30 for $s d$ less than $3 \mathrm{~cm}$.

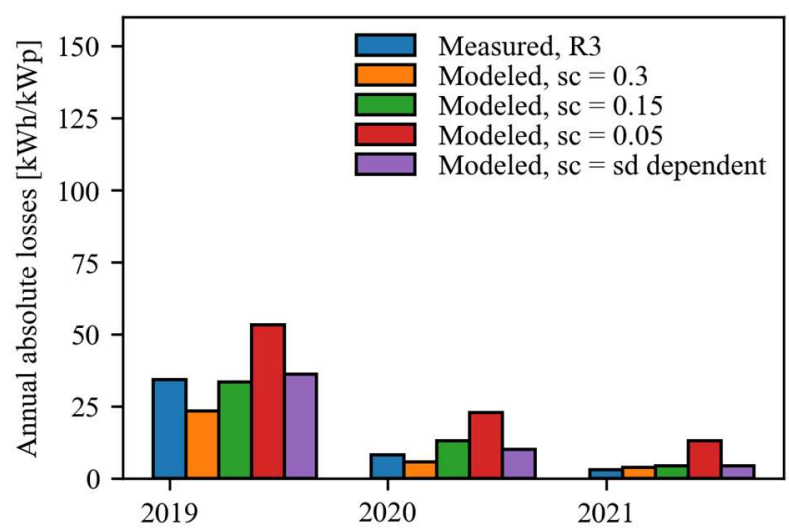

Fig.8. Measured and modeled annual absolute snow loss for the residential system with a tilt of $40^{\circ}$, located in an area with humid continental climate. The snow loss is modeled with varying $s c$ : $0.3,0.15,0.05$, and a snow depth $(s d)$ dependent $s c$, where $s c=0.05$ for $s d$ larger than $3 \mathrm{~cm}$ and 0.30 for $s d$ less than $3 \mathrm{~cm}$.

\section{CONCLUSION}

In this work we evaluate the Marion snow loss model with snow depth dependent snow clearing coefficients to account for the effect of different snow conditions on the natural snow clearing process. The model is tested using data from seven PV plants with two different system configurations, located in three different regions in Norway. We see that to achieve snow clearing coefficients that give satisfactory fit between measured and modeled losses for all years and for all systems with the same system configurations, snow depth dependency in the snow clearing coefficient has to be introduced. For the commercial systems some variations in the snow clearing rate between two of the systems were observed, related to variations in heat leakage from the roof below the systems. It was also observed that thin snow covers appear to introduce more uncertainty in the models compared to thicker snow covers.

\section{REFERENCES}

[1] IEA, "PVPS Trends in photovoltaic applications 2020", 2020.
[2] R.E. Pawluk, Y. Chen, Y. She, "Photovoltaic electricity generation loss due to snow - A literature review on influence factors, estimation, and mitigation", Renew. Sustain. Energy Rev., 107, pp. 171-182, 2019.

[3] B. Marion, R. Schaefer, H. Caine, G. Sanchez, "Measured and modeled photovoltaic system energy losses from snow for Colorado and Wisconsin locations", Sol. Energy, 97, pp. 112-121, 2013.

[4] D. Ryberg, J. Freeman, "Integration, validation, and application of a PV snow coverage model in SAM", Technical report: NREL/TP-6A20$68705,2017$.

[5] T. Townsend, L. Powers, "Photovoltaics and snow: An update from two winters of measurements in the Sierra", 37th IEEE Photovolt. Spec. Conf., pp. 3231-3236, 2011.

[6] E. Lorenz, D. Heinemann, C. Kurz, "Local and regional photovoltaic power prediction for large scale grid integration: Assessment of a new algorithm for snow detection", Prog. Photovoltaics Res. Appl., 20, pp. 760-769, 2011

[7] N. Heidari, J. Gwamuri, T. Townsend, J.M. Pearce, "Impact of snow and ground interference on photovoltaic electric system performance", IEEE J. Photovoltaics, 5, pp. 1680-1685, 2015.

[8] L. Burnham, D. Riley, J. Braid, "Design considerations for photovoltaic systems deployed in snowy climates", Proc. 37th Eur. Photovolt. Sol. Energy Conf. Exhib., pp. 1626-1631, 2020.

[9] M.B. Øgaard, B.L. Aarseth, Å.F. Skomedal, H.N. Riise, S. Sartori, J.H. Selj, "Identifying snow in photovoltaic monitoring data for improved snow loss modeling and snow detection”, Sol. Energy, 223, pp. 238-247, 2021.

[10] H. E. Beck, N. E. Zimmermann, T. R. McVicar, N. Vergopolan, A. Berg, E.F. Wood, "Present and future köppen-geiger climate classification maps at 1-km resolution", Sci. Data, 5, 2018.

[11] NVE, seNorge, 2019. www.senorge.no (accessed December 22, 2020).

[12] Norsk Klimaservicesenter, Observasjoner og værstatistikk, 2020. https://seklima.met.no/observations/ (accessed December 22, 2020).

[13] W.F. Holmgren, C.W. Hansen, M.A. Mikofski, "pvlib python: a python package for modeling solar energy systems," J. Open Source Softw., 3, 2018.

[14] A.F. Souka, H.H. Safwat, "Determination of the optimum orientations for the double exposure flat-plate collector and its reflections", Sol. Energy, 10, pp. 170-174, 1966.

[15] D. Riley, L. Burnham, B. Walker, J.M. Pearce, "Differences in snow shedding in photovoltaic systems with framed and frameless modules", 46th IEEE Photovolt. Spec. Conf., pp. 558-561, 2019.

[16] M. Ross, "Snow and ice accumulation on photovoltaic arrays: An assessment of the TN conseil passive melting technology", Division Report EDRL 95-68 (TR), 1995. 